\title{
Approximation of Zonoids by Zonotopes in Fixed Directions
}

\author{
S. Campi, ${ }^{1}$ D. Haas, ${ }^{2}$ and W. Weil ${ }^{3}$
}

Communicated by Rolf Schneider

\author{
${ }^{1}$ Dipartimento di Matematica "U. Dini," Università di Firenze, \\ Viale Morgagni 67A, 50134 Firenze, Italy \\ campi@udini.math.unifi.it \\ ${ }^{2}$ Berliner Strasse 286, 6050 Offenbach, Federal Republic of Germany \\ ${ }^{3}$ Mathematisches Institut II, Universität Karlsruhe, \\ Englerstrasse 2, 7500 Karlsruhe, Federal Republic of Germany \\ ac03@dkauni2.bitnet
}

\begin{abstract}
A construction is given which, for any zonoid $Z \subset \mathbf{R}^{d}$ and any unit vectors $u_{1}, \ldots, u_{k}$, produces a zonotope $Z^{\prime}$ which is a sum of at most $k$ segments and has the same support values as $Z$ in the directions $u_{1}, \ldots, u_{k}$. Applications to an estimation problem for fiber processes are also given as well as some related results for convex bodies.
\end{abstract}

\section{Introduction}

A zonotope in $d$-dimensional euclidean space $\mathbf{R}^{d}$ is a finite vector sum of line segments. A zonoid is a convex body which is the limit of zonotopes in the Hausdorff metric. Zonotopes and zonoids have a center of symmetry and, without loss of generality, we assume throughout that this center is at the origin. Then the support function $h(Z, \cdot):=\max _{y \in Z}\langle\cdot, y\rangle$ of the zonoid $Z$ has the form

$$
h(Z, \cdot)=\int_{\Omega}|\langle\cdot, x\rangle| \rho_{Z}(d x)
$$

with a (uniquely determined) even Borel measure $\rho_{Z}$ on the unit sphere $\Omega \subset \mathbf{R}^{d}$. 
Here $\langle\cdot, \cdot\rangle$ denotes the usual scalar product in $\mathbf{R}^{d}$. The measure $\rho_{Z}$ is called the generating measure of $Z$. For a zonotope $Z$, the generating measure $\rho_{Z}$ is supported by $k$ pairs of antipodal points and hence the integral in (1) reduces to a sum

$$
h(Z, \cdot)=\sum_{i=1}^{k}\left|\left\langle\cdot, x_{i}\right\rangle\right|
$$

with $x_{i} \in \mathbf{R}^{d}$.

The approximation of a given zonoid by zonotopes (e.g., with a fixed number of segments) has been discussed by a number of authors (Figiel et al., 1977; Betke and McMullen, 1983; Gordon, 1985; Schechtman, 1987; Bourgain and Lindenstrauss, 1988; Bourgain et al., 1989; Linhart, 1989). Zonoids and zonotopes have also found some interest in a number of practical problems in stochastic geometry. In particular, in the analysis of spatial fiber systems, observations are often of the form $h_{1}:=h\left(Z, \pm u_{1}\right), \ldots, h_{k}:=h\left(Z, \pm u_{k}\right)$ for some zonoid $Z \subset \mathbf{R}^{d}$ which is in general not explicitly known. The statistical problem is to give an estimate for the measure $\rho_{Z}$ on the basis of the observed values $h_{1}, \ldots, h_{k}$. A discrete solution to this problem is suggested by the following result.

Theorem 1. For a zonoid $Z \subset \mathbf{R}^{d}$ and unit vectors $u_{1}, \ldots, u_{k}$ there always exists a zonotope $Z^{\prime}$ which is the sum of at most $k$ segments and fulfills

$$
h\left(Z, \pm u_{1}\right)=h\left(Z^{\prime}, \pm u_{1}\right), \ldots, h\left(Z, \pm u_{k}\right)=h\left(Z^{\prime}, \pm u_{k}\right)
$$

As J. Bourgain pointed out to us, Theorem 1 can be obtained as a simple consequence of Carathéodory's theorem. For the above-mentioned application, however, a constructive approach is necessary which is not provided using Carathéodory's result. In the next section we therefore present an alternative proof of Theorem 1, which allows us to construct the zonotope $Z^{\prime}$ explicitly as the solution of a linear program. In Section 3 some background information on the stereological questions is given and the method of Section 2 is applied to the statistical problem mentioned above. The final section contains some related results which are consequences of either Theorem 1 or our method of proof.

Throughout the following we use some properties of zonoids and zonotopes without giving all the relevant details. Here we refer to the survey articles of Schneider and Weil (1983) and Goodey and Weil (1993). As references for basic notions from the theory of convex bodies, we mention Bonnesen and Fenchel (1934).

\section{Proofs of Theorem 1}

We first mention the following short and elegant approach to Theorem 1, which is due to $\mathrm{J}$. Bourgain. 
We may assume that the generating measure $\rho_{Z}$ of the given zonoid $Z$ is normalized, $\rho_{Z}(\Omega)=1$. For fixed directions $u_{1}, \ldots, u_{k} \in \Omega$ let $A \subset \mathbf{R}^{k}$ be the set of all vectors $\left(h\left(Z, u_{1}\right), \ldots, h\left(Z, u_{k}\right)\right)$ where $Z$ runs through the zonoids in $\mathbf{R}^{d}$ with normalized generating measure. $A$ is convex and compact. The subset $B \subset A$ consisting of those vectors coming from line segments $Z$ is compact and connected (it is a continuous image of $\Omega$ ). Obviously, $A$ is the closed convex hull of $B$, but because $B$ is closed, $A=$ conv $B$. By Caratheodory's theorem and since $B$ is connected, any point in $A$ is a convex combination of (at most) $k$ points from $B$. For our given vector $\left(h\left(Z, u_{1}\right), \ldots, h\left(Z, u_{k}\right)\right)$ this implies the existence of vectors $x_{1}, \ldots, x_{k} \in \mathbf{R}^{d}$ (with length $\frac{1}{2}$ ) and positive weights $\alpha_{1}, \ldots, \alpha_{k}$ with $\alpha_{1}+\cdots+\alpha_{k}=1$ and such that

$$
h\left(Z, u_{j}\right)=\sum_{i=1}^{k} \alpha_{i}\left|\left\langle u_{j}, x_{i}\right\rangle\right|, \quad j=1, \ldots, k .
$$

If we denote by $[-x, x]$ the segment from $-x$ to $x$, the zonotope

$$
Z^{\prime}:=\sum_{i=1}^{k} \alpha_{i}\left[-x_{i}, x_{i}\right]
$$

therefore fulfills the assertion.

In order to describe our alternative and constructive approach we consider, for given $u_{1}, \ldots, u_{k} \in \Omega$, the corresponding orthogonal hyperplanes $E_{1}, \ldots, E_{k}$ through the origin. They divide $\mathbf{R}^{d}$ into $m \leq 2^{k}$ polyhedral cones $C_{1}, \ldots, C_{m}$. Let

$$
\omega_{1}:=C_{1} \cap \Omega, \quad \omega_{2}:=\left(C_{2} \backslash C_{1}\right) \cap \Omega, \quad \omega_{3}:=\left(C_{3} \backslash\left(C_{1} \cup C_{2}\right)\right) \cap \Omega, \quad \ldots,
$$

thus $\omega_{1}, \ldots, \omega_{m}$ are spherically convex, pairwise disjoint Borel sets which cover $\Omega$.

For an arbitrary finite Borel measure $\rho$ on $\Omega$, let

$$
s_{i}:=\int_{\omega_{i}} x \rho(d x)
$$

be the centroid of the measure $\rho$ restricted to $\omega_{i}$ and define the zonotope $Z^{\prime \prime}$ as

$$
Z^{\prime \prime}:=\sum_{i=1}^{m}\left[-s_{i}, s_{i}\right]
$$

Then

$$
h\left(Z^{\prime \prime}, u_{j}\right)=\sum_{i=1}^{m}\left|\left\langle u_{j}, s_{i}\right\rangle\right|=\sum_{i=1}^{m}\left|\left\langle u_{j}, \int_{\omega_{1}} x \rho(d x)\right\rangle\right|=\sum_{i=1}^{m}\left|\int_{\omega_{1}}\left\langle u_{j}, x\right\rangle \rho(d x)\right| .
$$


On each $\omega_{i}$, the sign of $\left\langle u_{j}, \cdot\right\rangle$ does not change. Hence

$$
\left|\int_{\omega_{i}}\left\langle u_{j}, x\right\rangle \rho(d x)\right|=\int_{\omega_{i}}\left|\left\langle u_{j}, x\right\rangle\right| \rho(d x), \quad i=1, \ldots, m,
$$

and thus

$$
h\left(Z^{\prime \prime}, u_{j}\right)=\sum_{i=1}^{m} \int_{\omega_{t}}\left|\left\langle u_{j}, x\right\rangle\right| \rho(d x)=\int_{\Omega}\left|\left\langle u_{j}, x\right\rangle\right| \rho(d x)
$$

for $j=1, \ldots, k$.

In particular, for $\rho=\rho_{Z}$, we get $h\left(Z^{\prime \prime}, u_{1}\right)=h\left(Z, u_{1}\right), \ldots, h\left(Z^{\prime \prime}, u_{k}\right)=h\left(Z, u_{k}\right)$. The corresponding equations $h\left(Z^{\prime \prime},-u_{1}\right)=h\left(Z,-u_{1}\right), \ldots, h\left(Z^{\prime \prime},-u_{k}\right)=h\left(Z,-u_{k}\right)$ follow immediately from the symmetry of $Z$ and $Z^{\prime \prime}$. The number $m$ of segments of the zonotope $Z^{\prime \prime}$ is bounded above by Schläfli's lemma,

$$
m \leq 2 \sum_{r=0}^{d-1}\left(\begin{array}{c}
k-1 \\
r
\end{array}\right)
$$

but in general $m$ is greater than $k$. Therefore we now consider, for $\alpha_{1} \geq 0, \ldots$, $\alpha_{m} \geq 0$, the zonotope

$$
Z_{\alpha}:=\sum_{i=1}^{m} \alpha_{i}\left[-s_{i}, s_{i}\right]
$$

Let

$$
c_{i j}:=\left|\left\langle u_{j}, s_{i}\right\rangle\right|, \quad i=1, \ldots, m, j=1, \ldots, k
$$

then

$$
h\left(Z_{\alpha}, u_{j}\right)=\sum_{i=1}^{m} \alpha_{i} c_{i j}, \quad j=1, \ldots, k
$$

The optimization problem of choosing $\alpha_{1}, \ldots, \alpha_{m}$ so as to minimize the distance between $Z_{\alpha}$ and $Z$ in the given directions $u_{1}, \ldots, u_{k}$ can be formulated as a linear program:

$$
\begin{aligned}
& \text { Minimize } f\left(\alpha_{1}, \ldots, \alpha_{m}\right):=\sum_{j=1}^{k}\left(h_{j}-\sum_{i=1}^{m} \alpha_{i} c_{i j}\right) \\
& \text { subject to } \sum_{i=1}^{m} \alpha_{i} c_{i j} \leq h_{j}, \quad j=1, \ldots, k ; \quad \alpha_{i} \geq 0, \quad i=1, \ldots, m .
\end{aligned}
$$


Introducing slack variables $y_{1}, \ldots, y_{k}$, the set of feasible solutions is transformed into the region $M \subset \mathbf{R}^{m} \times \mathbf{R}^{k}$ given by

$$
\begin{gathered}
\sum_{i=1}^{m} \alpha_{i} c_{i j}+y_{j}=h_{j}, \quad j=1, \ldots, k \\
\alpha_{i} \geq 0, \quad y_{j} \geq 0, \quad i=1, \ldots, m, \quad j=1, \ldots, k .
\end{gathered}
$$

Since the objective function $\tilde{f}, \tilde{f}\left(\alpha_{1}, \ldots, \alpha_{m}, y_{1}, \ldots, y_{k}\right):=f\left(\alpha_{1}, \ldots, \alpha_{m}\right)$, is bounded below by 0 on $M$, the transformed linear program is solvable, and in fact a solution is given by $\alpha_{1}=\cdots=\alpha_{m}=1, y_{1}=\cdots=y_{k}=0$ with minimal value $\tilde{f}=0$, as we have seen above. A standard result from optimization theory (see, e.g., Karlin, 1959) says that there is also a vertex $\left(\alpha_{1}, \ldots, \alpha_{m}, y_{1}, \ldots, y_{k}\right) \in M$ which solves the problem, hence has $\tilde{f}\left(\alpha_{1}, \ldots, \alpha_{m}, y_{1}, \ldots, y_{k}\right)=0$. Moreover, if we write the constraints

$$
\sum_{i=1}^{m} \alpha_{i} c_{i j}+y_{j}=h_{j}, \quad j=1, \ldots, k
$$

in matrix form,

$$
A\left(\begin{array}{l}
\alpha \\
y
\end{array}\right)=h
$$

with $\alpha=\left(\alpha_{1}, \ldots, \alpha_{m}\right)^{\mathrm{T}}, y=\left(y_{1}, \ldots, y_{k}\right)^{\mathrm{T}}, h=\left(h_{1}, \ldots, h_{k}\right)^{\mathrm{T}}$, and

$$
A=\left(C \mid E_{k}\right)
$$

(where $C=\left(\left(c_{i j}\right)\right)$ and $E_{k}$ is the $k$ by $k$ identity matrix), then any vertex $v$ of $M$ has the property that the columns of $A$ which belong to the nonzero coordinates of $v$ are linearly independent. Since a vertex solution of our linear program must have $y=0$ and since the rank of $C$ is no more than $k$, we get that at most $k$ values of $\alpha_{1}, \ldots, \alpha_{m}$ in our solution are nonzero. The corresponding zonotope $Z^{\prime}:=Z_{\alpha}$ therefore consists of at most $k$ segments and satisfies

$$
\sum_{j=1}^{k}\left(h_{j}-\sum_{i=1}^{m} \alpha_{i} c_{i j}\right)=0
$$

(as well as $h_{j}-\sum_{i=1}^{m} \alpha_{i} c_{i j} \geq 0, j=1, \ldots, k$ ), hence

$$
h\left(Z^{\prime}, u_{j}\right)=h_{j}, \quad j=1, \ldots, k
$$




\section{Fiber Processes}

In the following stereological applications we concentrate on random collections of fibers (so-called fiber processes; mathematically these are point processes of curves). Random collections of lines, hyperplanes, or sheets (surfaces) can be treated in a similar way. We assume that the fiber process is stationary, i.e., its distribution is translation invariant. We cannot give all the relevant background here and refer to Stoyan et al. (1987) or Weil (1987) for more details.

The mean behavior of a stationary fiber process $X$ in $\mathbf{R}^{d}$ (with locally finite intensity measure) is described by two quantities, the length density $L_{V}$ and the directional distribution $P$. The first is a positive number which describes the mean total length of the fibers per unit volume; the second is an even probability measure on $\Omega$ which gives the distribution of the tangent direction in a typical point of the fibers. We remark that $L_{V}$ and $P$ do not depend on the individual fibers but only on their union set. Therefore, the fiber process $X$ may be also considered as a random one-dimensional closed set in $\mathbf{R}^{d}$ (this is, e.g., the point of view in Stoyan et al. (1987)). If $E \subset \mathbf{R}^{d}$ is a hyperplane (with a randomly chosen direction, independent of $X$ ), then the intersection field $X \cap E$ is almost surely a stationary process of ordinary points in $E$, the mean behavior of which is described by a single quantity, the intensity $\gamma_{E} \cdot \gamma_{E}$ gives the mean number of (intersection) points per unit $(d-1)$-dimensional volume in $E$. Again by stationarity, $\gamma_{E}$ depends only on the direction $\pm u$ of $E$, hence it gives rise to an even function $h$ on $\Omega, h(u):=\gamma_{u^{+}}$. The following integral equation connects $h, L_{V}$, and $P$ :

$$
h=L_{V} \int_{\Omega}|\langle\cdot, x\rangle| P(d x) .
$$

Thus, there is a unique zonoid $Z=Z(X)$ associated with the fiber process $X$. The generating measure of $Z$ is given by $\rho_{Z}:=L_{V} \cdot P$, and the support function $h(Z, \cdot)$ of $Z$ describes the intersection intensities of $X$.

The estimation problem mentioned in the Introduction occurs in applications in biology, medicine, or geology in the following way: The (three-dimensional) fiber process $X$ can only be observed in finitely many planar sections $X \cap E_{1}, \ldots$, $X \cap E_{k}$ and the mean number of intersection points (per unit area) can be counted in each sectioning plane. Thus, (unbiased) estimators for the intensities $\gamma_{E_{1}}, \ldots, \gamma_{E_{k}}$ can be given. The problem is then to estimate the measure $\rho_{Z}$ from these values (which in turn gives estimators for $L_{V}=\rho_{Z}(\Omega)$ and $P=\rho_{Z} / \rho_{Z}(\Omega)$ ). A discrete approximation (and estimation) of $\rho_{Z}$ is given by the generating measure $\rho_{Z^{\prime}}$ of a zonotope which best fits the values $\gamma_{E_{1}}=h\left(Z, u_{1}\right), \ldots, \gamma_{E_{k}}=h\left(Z, u_{k}\right)$ (with $u_{i} \perp E_{i}$ ). The existence of such a zonotope $Z^{\prime}$ is expressed by Theorem 1 and the method of the last section shows how to construct $Z^{\prime}$. However, the procedure has to be modified slightly, since up to now the construction of $Z^{\prime}$ requires the knowledge of $\rho_{Z}$.

As before, let $C_{1}, \ldots, C_{m}$ be the closed polyhedral cones which are generated by the hyperplanes $u_{1}^{\perp}, \ldots, u_{k}^{\perp}$. For an interior point $y \in C_{i}$ and $j \in\{1, \ldots, k\}$, let $\varepsilon_{i j}$ 
be the sign of $\left\langle u_{j}, y\right\rangle$. We consider the zonotope

$$
Z\left(x_{1}, \ldots, x_{m}\right):=\sum_{i=1}^{m}\left[-x_{i}, x_{i}\right]
$$

where $x_{1}, \ldots, x_{m} \in \mathbf{R}^{d}$ are variable. Then, by the results of Section 2 , it follows that the linear program,

$$
\begin{array}{ll}
\text { Minimize } & f\left(x_{1}, \ldots, x_{m}\right):=\sum_{j=1}^{k}\left(h_{j}-\sum_{i=1}^{m} \varepsilon_{i j}\left\langle u_{j}, x_{i}\right\rangle\right) \\
\text { subject to } & \sum_{i=1}^{m} \varepsilon_{i j}\left\langle u_{j}, x_{i}\right\rangle \leq h_{j}, \quad j=1, \ldots, k, \\
& \text { and } x_{i} \in C_{i}, \quad i=1, \ldots, m,
\end{array}
$$

is solvable, and a solution is given by

$$
x_{1}=s_{1}, \ldots, x_{m}=s_{m},
$$

with optimal value

$$
f\left(x_{1}, \ldots, x_{m}\right)=0
$$

This linear program has $m d$ variables and $(m+1) k$ inequality constraints, since the condition $x_{i} \in C_{i}$ can be replaced by $k$ inequalities

$$
\varepsilon_{i j}\left\langle u_{j}, x_{i}\right\rangle \geq 0, \quad j=1, \ldots, k
$$

The method described here is valid for each dimension $d \geq 2$, but only of practical interest in the three-dimensional case. For planar fiber fields $X$, the estimation of the directional distribution is quite often also not done directly, but with the help of the intersection point fields on random lines. The determination of the intensity of the latter is much less problematic than the direct measurement of tangential directions. The fitting of a zonotope $Z^{\prime}$ to the support values $h_{1}, \ldots, h_{k}$ of the underlying unknown zonoid $Z$ is however much simpler in the planar case since in $\mathbf{R}^{2}$ every centrally symmetric polygon is a zonotope. Hence the corresponding optimization problem can be easily solved by the zonotope

$$
Z^{\prime}=\bigcap_{i=1}^{k}\left\{x \in \mathbf{R}^{2}:\left\langle u_{i}, x\right\rangle \leq h_{i}\right\},
$$

and the corresponding estimator for $\rho_{Z}$ is obtained from the edge lengths of $Z^{\prime}$. This procedure was proposed in Weil (1987) and has been discussed in more detail in Rataj and Saxl (1989). 
We finally mention that the estimation procedures discussed here are consistent, if the directions in Theorem 1 are chosen randomly (i.e., independently and with uniform distribution). To be more precise, let $Z \subset \mathbf{R}^{d}$ be a zonoid, let $u_{1}, u_{2}, \ldots$ be a sequence of independent and uniformly distributed random vectors in $\Omega$, and let $Z^{(k)}$ be the zonotope which exists by Theorem 1 for the first $k$ elements $u_{1}, \ldots, u_{k}$ of this sequence (to be more precise, we should say one polytope, because in general $Z^{(k)}$ may not be uniquely determined). Then the random zonotope $Z^{(k)}$ converges almost surely to $Z$, as $k \rightarrow \infty$.

This follows easily, since the sequence $Z^{(1)}, Z^{(2)}, \ldots$ is bounded, and we may therefore assume, by the Blaschke Selection Theorem, that $Z^{(1)}, Z^{(2)}, \ldots$ converge to a zonoid $\tilde{Z}$. Then $\tilde{Z}$ satisfies

$$
h\left(\tilde{Z}, u_{i}\right)=h\left(Z, u_{i}\right), \quad i=1,2, \ldots
$$

Since the sequence $u_{1}, u_{2}, \ldots$ is almost surely dense in $\Omega$, the continuous functions $h(Z, \cdot)$ and $h(Z, \cdot)$ coincide on a dense subset of $\Omega$. Therefore, $h(Z, \cdot)=h(Z, \cdot)$ and thus $\tilde{Z}=Z$.

The convergence of $Z^{(k)}$ to $Z$ implies the weak convergence of $\rho_{Z^{(k)}}$ to $\rho_{Z}$. Therefore, if $Z=Z(X)$ is the associated zonoid of a fiber process $X$, the estimated (discrete) directional distributions $P^{(k)}:=\rho_{Z^{(k)} / \rho_{Z^{(k)}}(\Omega)}$ converge almost surely (and weakly) to the directional distribution $P$ of $X$.

This result holds similarly for sequences $u_{1}, u_{2}, \ldots$ on $\Omega$ which are uniformly distributed in the sense of number theory (for more details, see Linhart, 1989).

\section{Related Results}

In the following we collect some further results which are related to Theorem 1 or follow from the construction in Section 2. First, we consider similar results for projections, which stem from the interpretation of zonoids as projection bodies.

The class of zonoids in $\mathbf{R}^{d}$ with inner points coincides with the class of projection bodies (of bodies with inner points). For a convex body $K \subset \mathbf{R}^{d}$ without inner points, the projection body is either a segment (if $K$ is $(d-1)$-dimensional) or a point (if $K$ is at most $(d-2)$-dimensional). To be more precise, let $v(K, u)$ denote the $(d-1)$-dimensional content of the orthogonal projection of the convex body $K$ onto the hyperplane orthogonal to $u,\|u\|=1$. Then

$$
v(K, u)=\frac{1}{2} \int_{\Omega}|\langle u, x\rangle| S_{d-1}(K, d x)
$$

where $S_{d-1}(K, \cdot)$ denotes the $(d-1)$ st surface area measure of $K$. The projection body $\Pi K$ of $K$ is then defined by $h(\Pi K, \cdot):=v(K, \cdot)$. $\Pi K$ is a zonoid with generating measure $\frac{1}{4}\left(S_{d-1}(K, \cdot)+S_{d-1}\left(K^{*}, \cdot\right)\right)$ (here, $K^{*}$ denotes the reflection of $K$ with respect to the origin). 
In the following proof we make use of the mixed volume

$$
V_{1}(K, L):=V(K, \ldots, K, L)=\frac{1}{d} \int_{\Omega} h(L, x) S_{d-1}(K, d x)
$$

for convex bodies $K, L$, and of the Minkowski inequality

$$
V^{d-1}(K) V(L) \leq V_{1}^{d}(K, L)
$$

(here $V$ denotes the volume), where equality holds for bodies $K, L$ with inner points, if and only if $K$ and $L$ are homothetic.

Proposition 2. Let $K$ be a convex body with inner points, which is not a polytope, and let $u_{1}, \ldots, u_{k}$ be arbitrary directions. Then there exists a polytope $P$ with

$$
v\left(K, \pm u_{1}\right)=v\left(P, \pm u_{1}\right), \ldots, v\left(K, \pm u_{k}\right)=v\left(P, \pm u_{k}\right)
$$

and such that $V(P)<V(K)$.

Proof. Let $Z:=\Pi K$ and $Z^{\prime \prime}$ be the zonotope constructed in Section 2 for the measure $\rho=S_{d-1}(K, \cdot)$. Let $\rho^{\prime}$ be the discrete measure on $\Omega$, defined by $\rho^{\prime}\left(s_{i} /\left\|s_{i}\right\|\right):=\left\|s_{i}\right\|$, for all $i \in\{1, \ldots, m\}$ with $s_{i} \neq 0$. $\rho^{\prime}$ has centroid 0 since

$$
\int_{\Omega} x \rho^{\prime}(d x)=\sum_{i=1}^{m} s_{i}=\sum_{i=1}^{m} \int_{\omega_{i}} x \rho(d x)=\int_{\Omega} x S_{d-1}(K, d x)=0
$$

If $Z^{\prime \prime}$ has inner points, then, by Minkowski's existence theorem, $\rho^{\prime}$ is the surface area measure of a polytope $P$ which fulfills $Z^{\prime \prime}=\Pi P$ and therefore (5) (in view of (2)).

Moreover, we have, from (3) and the convexity of $h(K, \cdot)$,

$$
\begin{aligned}
V_{1}(P, K) & =\frac{1}{d} \int_{\Omega} h(K, x) S_{d-1}(P, d x)=\frac{1}{d} \int_{\Omega} h(K, x) \rho^{\prime}(d x) \\
& =\frac{1}{d} \sum_{i=1}^{m} h\left(K, s_{i}\right)=\frac{1}{d} \sum_{i=1}^{m} h\left(K, \int_{\omega_{i}} x \rho(d x)\right) \\
& \leq \frac{1}{d} \sum_{i=1}^{m} \int_{\omega_{i}} h(K, x) S_{d-1}(K, d x)=\frac{1}{d} \int_{\Omega} h(K, x) S_{d-1}(K, d x)=V(K)
\end{aligned}
$$


Thus, (4) implies

$$
V^{d-1}(P) V(K) \leq V^{d}(K)
$$

and hence

$$
V(P) \leq V(K)
$$

with equality, if and only if $P$ and $K$ are translates.

If $Z^{\prime \prime}$ has no inner points, we choose additional directions $u_{k+1}, \ldots, u_{\mathrm{r}}$ and consider the corresponding zonotope $Z^{r}$ which satisfies

$$
h\left(Z, u_{1}\right)=h\left(Z^{r}, u_{1}\right), \ldots, h\left(Z, u_{r}\right)=h\left(Z^{r}, u_{r}\right)
$$

Since $Z$ has inner points, we can make this choice in such a way that $Z^{r}$ also has inner points. By the above arguments, the corresponding polytope $P^{r}$ then fulfills the assertion and the proof is completed.

The result has an interesting consequence for the following problem. For a given body $K$ and directions $u_{1}, \ldots, u_{k}$ let $\mathscr{A}$ be the set of all convex bodies $L$ with

$$
v\left(K, \pm u_{1}\right)=v\left(L, \pm u_{1}\right), \ldots, v\left(K, \pm u_{k}\right)=v\left(L, \pm u_{k}\right)
$$

If there is an element $L_{\min } \in \mathscr{A}$ which has minimal volume $V\left(L_{\text {min }}\right)>0$, then $L_{\text {min }}$ is a polytope. In general, it seems to be an open problem to determine whether or under which conditions minimal or maximal elements in $\mathscr{A}$ do exist and to characterize them.

It is more complicated to formulate a result, analogous to Proposition 2, where the volume condition is replaced by a condition on the number of facets of $P$. The reason is that the zonotope $Z^{\prime}$ from Section 2 need not be a projection body, even if the zonoid $Z=\Pi K$ is smooth and the directions $u_{1}, \ldots, u_{k}$ are in general position. In case $2 \leq \operatorname{dim} Z^{\prime} \leq d-1$ we have to proceed as in the proof of Proposition 1 and add $r:=d-\operatorname{dim} Z^{\prime}$ suitable directions $u_{k+1}, \ldots, u_{k+r}$. As a result we get, for example, a centrally symmetric polytope $P$ with at most $k+d-2$ pairs of opposite facets and such that

$$
v\left(K, \pm u_{1}\right)=v\left(P, \pm u_{1}\right), \ldots, v\left(K, \pm u_{k}\right)=v\left(P, \pm v_{k}\right)
$$

Another direct consequence of Theorem 1 concerns an illumination problem posed by Martini (1987). For the case that $Z$ is the unit ball and arbitrary directions $u_{1}, \ldots, u_{k}$, the theorem implies that there is a zonotope $Z^{\prime}$ which is the sum of (at most) $k$ segments and fulfills

$$
h\left(Z^{\prime}, u_{1}\right)=1, \ldots, h\left(Z^{\prime}, u_{k}\right)=1
$$


In terms of parallel illumination this implies that the whole boundary of an arbitrary convex polytope $P \subset \mathbf{R}^{d}$ with $k$ facets can be isophotically illuminated by $k$ parallel illuminations (see Martini, 1987, for more details).

Finally, we get a solution to a problem on zonoids, which was posed in a slightly less general form by the last author at the Oberwolfach meeting on "Convex Geometry" in 1982.

Proposition 3. Let $Z \subset \mathbf{R}^{d}$ be a zonoid and let $P, Q \subset \mathbf{R}^{d}$ be polytopes with

$$
P \subset Z \subset Q
$$

Then there is a sequence $\left(Z_{k}\right)_{k \in N}$ of zonotopes $Z_{k}$ with $Z_{k} \rightarrow Z($ as $k \rightarrow \infty)$ and

$$
P \subset Z_{k} \subset Q, \quad k \in \mathbf{N}
$$

Proof. Without loss of generality, we may assume that $P, Z$, and $Q$ have interior points and that all vertices $x_{1}, \ldots, x_{r}$ of $P$ lie on the boundary of $Z$ (the latter follows if we replace $P$ by an appropriate larger polytope).

We first consider the case, where $Z$ is strictly convex and smooth (i.e., each boundary point has a unique supporting hyperplane) and $Z$ lies in the interior, int $Q$, of $Q$. Let $u_{1}, \ldots, u_{r}$ be the (uniquely determined) outer normals of $Z$ at the points $x_{1}, \ldots, x_{r}$. We choose $\varepsilon>0$ such that, for $1 \leq i \leq r$, the ball $B_{i}$ of radius $\varepsilon$ centered at $u_{i}$ lies in the interior of the normal cone of $P$ at the vertex $x_{i}$. Then there are (linearly independent) unit vectors $u_{i j}, j=1, \ldots, d$, in $B_{i}$ such that $u_{i}$ lies in the interior of the cone spanned by $u_{i 1}, \ldots, u_{i d}$. Let $v_{1}, \ldots, v_{s}$ be the facet normals of $Q$ and define $h_{i j}:=h\left(Z, u_{i j}\right), i=1, \ldots, r, j=1, \ldots, d, h_{i 0}:=h\left(Z, u_{i}\right), i=1, \ldots, r$, and $\tilde{h}_{k}:=h\left(Z, v_{k}\right), k=1, \ldots, s$. We define the polytope

$$
\begin{aligned}
R_{\varepsilon} & :=\bigcap_{i=1}^{r} \bigcap_{j=1}^{d}\left\{x \in \mathbf{R}^{d}:\left\langle u_{i j}, x\right\rangle \leq h_{i j}\right\} \\
& \cap \bigcap_{i=1}^{r}\left\{x \in \mathbf{R}^{d}:\left\langle u_{i}, x\right\rangle \leq h_{i 0}\right\} \cap \bigcap_{k=1}^{s}\left\{x \in \mathbf{R}^{d}:\left\langle v_{k}, x\right\rangle \leq \tilde{h}_{k}\right\} .
\end{aligned}
$$

Then, obviously, $Z \subset R_{\varepsilon} \subset Q$. Moreover, the polytope $R_{\varepsilon}$ is circumscribed to $Z$ and since $Z$ is strictly convex and smooth, the unit vectors $u_{i}, u_{i j}, v_{k}, i=1, \ldots, r$, $j=1, \ldots, d, k=1, \ldots, s$, are exactly the facet normals of $R_{\varepsilon}$. The facet $R_{\varepsilon}\left(u_{i}\right)$ in direction $u_{i}$ contains $x_{i}$ and, for $\varepsilon \rightarrow 0, R_{\varepsilon}\left(u_{i}\right)$ shrinks to the point $x_{i}$. By Theorem 1 , there is a zonotope $Z_{\varepsilon}$ with at most $m:=r(d+1)+s$ segments which fulfills

$$
h\left(Z_{\varepsilon}, u_{i j}\right)=h_{i j}, \quad i=1, \ldots, r, j=0, \ldots, d,
$$

and

$$
h\left(Z_{\varepsilon}, v_{k}\right)=\tilde{h}_{k}, \quad k=1, \ldots, s
$$


By construction, we have $Z_{\varepsilon} \subset R_{\varepsilon} \subset Q$ and $Z_{\varepsilon}$ has a vertex in every facet of $R_{\varepsilon}$. In particular, there is a point of $Z_{\varepsilon}$ in $R_{\varepsilon}\left(u_{i}\right), i=1, \ldots, r$. Blaschke's Selection Theorem implies that we can choose a sequence $\varepsilon_{n} \rightarrow 0$ such that the zonotopes $Z_{\varepsilon_{n}}$ converge, as $n \rightarrow \infty$, to a convex body $Z^{\prime}$. Since all zonotopes $Z_{\varepsilon_{n}}$ have at most $m$ segments, $Z^{\prime}$ has to be a zonotope with the same property. Moreover, since the facets $R_{\varepsilon_{n}}\left(u_{i}\right)$ shrink to $x_{i}$, all vertices $x_{1}, \ldots, x_{r}$ of $P$ must lie in $Z^{\prime}$. Therefore, $P \subset Z^{\prime} \subset Q$.

Now we consider the general case. First we still assume that $Z$ is in the interior of $Q$, but not necessarily strictly convex and smooth. By a familiar smoothing procedure, $Z$ can be approximated from outside by strictly convex and smooth zonoids $Z_{\eta}, Z_{\eta} \rightarrow Z$ as $\eta \rightarrow 0$, and such that $Z_{\eta} \subset Q$. From the argument above, we get a zonotope $Z_{\eta}^{\prime}$ with $P \subset Z_{\eta}^{\prime} \subset Q$ for each $\eta>0$. Since all $Z_{\eta}^{\prime}$ have at most $m$ segments we again get, for $\eta \rightarrow 0$, a zonotope $Z^{\prime}$ with the property $P \subset Z^{\prime} \subset Q$. The restriction $Z \subset$ int $Q$ can now be removed in a similar manner, e.g., by approximating $Q$ from outside by $\rho Q, \rho>1$.

We have now shown that there is a zonotope $Z^{\prime}$ with

$$
P \subset Z^{\prime} \subset Q
$$

The proof of Proposition 3 is thus completed if we replace $P$ by an increasing sequence of polytopes and $Q$ by a decreasing sequence, both converging to $Z$.

\section{References}

Betke, U., McMullen, P., Estimating the sizes of convex bodies from projections, J. London Math. Soc. 27 (1983), 525-538.

Bonnesen, T., Fenchel, W., Theorie der konvexen Körper, Springer-Verlag, Berlin, 1934.

Bourgain, J., Lindenstrauss, J., Distribution of points on spheres and approximation by zonotopes, Israel J. Math. 64 (1988), 25-31.

Bourgain, J., Lindenstrauss, J., Milman, V., Approximation of zonoids by zonotopes, Acta Math. 162 (1989), 73-141.

Figiel, T., Lindenstrauss, J., Milman, V., The dimension of almost spherical sections of convex bodies, Acta Math. 139 (1977), 53-94.

Goodey, P., Weil, W., Zonoids and generalisations, in: Handbook of Convex Geometry (P. Gruber and J. M. Wills, eds.), Elsevier Science, Amsterdam, 1993, in print.

Gordon, Y., Some inequalities for Gaussian processes and applications, Israel J. Math. 50 (1985), 265-289.

Karlin, S., Mathematical Methods and Theory in Games, Programming and Economics, Vols. I and II, Pergamon, London, 1959.

Linhart, J., Approximation of a ball by zonotopes using uniform distribution on the sphere, Arch. Math. 53 (1989), 82-86.

Martini, H., Some results and problems around zonotopes, Intuitive Geometry (Siofok, 1985), Colloquia Mathematica Societatis János Bolyai, Vol. 48, North-Holland, Amsterdam, 1987, pp. 383-417.

Rataj, J., Saxl, I., Analysis of planar anisotropy by means of the Steiner compact, J. Appl. Probab. 26 (1989), 490-502

Schechtman, G., More on embedding subspaces of $L_{p}$ in $l_{p}^{m}$, Compositio Math. 61 (1987), 159-170. 
Schneider, R., Weil, W., Zonoids and related topics, in: Convexity and Its Applications (P. Gruber and J. M. Wills, eds.), Birkhäuser, Basel, 1983, pp. 296-317.

Stoyan, D., Kendall, W. S., Mecke, J., Stochastic Geometry and Its Applications, Akademie-Verlag, Berlin, Wiley, New York, 1987.

Weil, W., Point processes of cylinders, particles, and flats, Acta Appl. Math. 9 (1987), 103-136.

Received September 22, 1992. 